\title{
The Convergence and MS Stability of Exponential Euler Method for Semilinear Stochastic Differential Equations
}

\author{
Chunmei Shi, Yu Xiao, and Chiping Zhang \\ Department of Mathematics, Harbin Institute of Technology, Harbin 150001, China \\ Correspondence should be addressed to Yu Xiao, yxiao@hit.edu.cn
}

Received 23 May 2012; Accepted 4 June 2012

Academic Editor: Yeong-Cheng Liou

Copyright (C) 2012 Chunmei Shi et al. This is an open access article distributed under the Creative Commons Attribution License, which permits unrestricted use, distribution, and reproduction in any medium, provided the original work is properly cited.

The numerical approximation of exponential Euler method is constructed for semilinear stochastic differential equations (SDEs). The convergence and mean-square (MS) stability of exponential Euler method are investigated. It is proved that the exponential Euler method is convergent with the strong order $1 / 2$ for semilinear SDEs. A mean-square linear stability analysis shows that the stability region of exponential Euler method contains that of EM method and stochastic Theta method $(0 \leq \theta<1)$ and also contains that of the scale linear SDE, that is, exponential Euler method is analogue mean-square $A$-stable. Then the exponential stability of the exponential Euler method for scalar semi-linear SDEs is considered. Under the conditions that guarantee the analytic solution is exponentially stable in mean-square sense, the exponential Euler method can reproduce the mean-square exponential stability for any nonzero stepsize. Numerical experiments are given to verify the conclusions.

\section{Introduction}

Stochastic differential equations are utilized as mathematical models for physical application that possesses inherent noise and uncertainty. Such models have played an important role in a range of applications, including biology, chemistry, epidemiology, microelectronics, and finance. Many mathematicians have devoted their effort to develop it and have obtained a substantial body of achievements. In order to understand the dynamics of stochastic system, it is important to construct an efficient numerical simulation of SDEs. There are many results for numerical solutions of stochastic differential equations. The general introduction to numerical methods for SDEs can be found in [1-3].

The related concepts of $p$ th moment stability $(0<p \leq 2)$ are attractive in its own right for analytical solution and numerical solution (see $[4,5])$. Especially, MS stability $(p=2)$ is 
considered in a vast array of the literature $[6,7]$. The mean-square and almost sure asymptotic stability analysis of stochastic Theta method $(0 \leq \theta<1)$ for test equation are investigated in $[8,9]$.

The phenomenon of stiffness appears in the process of applying a certain numerical method to solve ODEs and SEDs. Stiffness was also the reason for the introduction of exponential integrators, which have been proposed independently by many authors. The main contribution of exponential integrators is that it can solve exactly the linear part of the problem [10]. Due to the cost of computing, the Jacobian, and the exponential or related function of Jacobian, many work was directed at the semi-linear problems.

Lawson [11] firstly combined the exponential function with explicit Runge-Kutta (RK) methods to obtain A-stability, as the well-known Lawson-Euler method aiming to overcome the stiffness in the semi-linear problems. The exponential RK numerical scheme was considered for the time integration of semi-linear parabolic problems in [12] and the numerical schemes allowed the construction of methods of arbitrarily high order with good stability properties [13]. In [14] sufficient conditions that guarantee exponential RK methods is contractive and asymptotically stable were given for semi-linear systems of ordinary differential equations. However, little is hitherto known about the convergence and stability of exponential RK methods when it is applied to SDEs.

In this paper, the exponential Euler method as one of the simplest forms of exponential RK method is extended to semi-linear SDEs. The exponential Euler method is based on a discrete version of the variation of constants formula.

In Section 2, the exponential Euler method is proposed to semi-linear SDEs and the convergence analysis of this method is investigated on any bounded interval $[0, T]$.

In Section 3.1, the scalar linear stochastic differential equation as test equation is used to calculate the MS stability region of exponential Euler method. One surprising observation is that the exponential Euler as a kind of explicit numerical method has a good stability property, that is, the MS stability region of this method contains that of the test equation and contains that of EM method and stochastic Theta method $(0 \leq \theta<1)$. In another word, if the test equation is MS stable, then so is the exponential Euler method applied to the systems for any stepsize. However, the classical explicit numerical methods such as EulerMaruyama (EM) method in [5, 15] and Milstein method [16] and the semi-explicit method such as stochastic Theta method $(0 \leq \theta<1 / 2)$ in $[8,9]$ usually have some limitations to the stepsize.

We then consider the exponentially MS stability of the exponential Euler method for scalar semi-linear SDEs in Section 3.2. It is proved that under the conditions that guarantee semi-linear SDEs are exponentially MS stable, the exponential Euler method can preserve stability property for any stepsize. Some numerical examples are provided in Section 4 to illustrate the theoretic results.

\section{Exponential Euler Method and Strong Convergence}

Throughout this paper, unless otherwise specified, let $|\cdot|$ be the Euclidean norm in $\mathbb{R}^{n}$. If $A$ is a vector or matrix, its transpose is denoted by $A^{T}$. If $A$ is a matrix, its trace norm is denoted by $|A|=\sqrt{\operatorname{trace}\left(A^{T} A\right)}$. Let $(\Omega, \mathcal{F}, \mathbb{P})$ be a complete probability space with a filtration $\left\{\mathcal{F}_{t}\right\}_{t \geq 0}$ satisfying the usual conditions, that is right continuous and increasing, while $\mathcal{F}_{0}$ contains all $p$-null sets. Let $W(t)$ be a scalar Wiener process defined on the probability space. Let $p>0$ and $L_{\mathscr{f}_{0}}^{p}\left(\Omega ; \mathbb{R}^{n}\right)$ denote the family of $\mathbb{R}^{n}$-valued $\mathscr{F}_{0}$-measurable random variables $\xi$ with $\mathbb{E}|\xi|^{p}<\infty$. 


\subsection{Exponential Euler Method}

We consider the $n$-dimensional semi-linear SDEs

$$
\begin{aligned}
d X(t) & =F X(t)+f(t, X(t)) d t+g(t, X(t)) d W(t), \\
X(0) & =X_{0},
\end{aligned}
$$

where initial data $X_{0} \in L_{\mathscr{f}_{0}}^{p}\left(\Omega ; R^{n}\right), F \in \mathbb{R}^{n \times n}$ is the generator of a strongly continuous analytic semigroup $S=(S(t))_{t \geq 0}$ on a Banach space [17], $f:[0, T] \times \mathbb{R}^{n} \rightarrow \mathbb{R}^{n}, g:[0, T] \times \mathbb{R}^{n} \rightarrow \mathbb{R}^{n}$ and $W(t)$ is a scalar Wiener process.

In our analysis, it will be more natural to work with the equivalent expression

$$
X(t)=e^{F t} X_{0}+\int_{0}^{t} e^{F(t-s)} f(s, X(s)) d s+\int_{0}^{t} e^{F(t-s)} g(s, X(s)) d W(s) .
$$

Now, we introduce the exponential Euler method for (2.1). Given a stepsize $h>0$, the exponential Euler approximate solution is defined by

$$
y_{k+1}=e^{F h} y_{k}+e^{F h} f\left(t_{k}, y_{k}\right) h+e^{F h} g\left(t_{k}, y_{k}\right) \Delta W_{k}
$$

where $y_{k}$ is an approximation to $X\left(t_{k}\right)$ with $t_{k}=k h, y_{0}=X_{0}$ and $\Delta W_{k}=W\left(t_{k+1}\right)-W\left(t_{k}\right)$ is the Wiener increment. It is convenient to use the continuous exponential Euler approximate solution and hence $y(t)$ is defined by

$$
y(t):=e^{F t} y_{0}+\int_{0}^{t} e^{F(t-\bar{s})} f(\bar{s}, Y(s)) d s+\int_{0}^{t} e^{F(t-\bar{s})} g(\bar{s}, Y(s)) d W(s),
$$

where $\bar{s}=[s / h] h$ and $[x]$ denote the largest integer, which is smaller than $x$ and $Y(t)$ is the step function which defined by

$$
Y(t):=\sum_{k=0}^{\infty} I_{\left[t_{k}, t_{k+1}\right)}(t) y_{k}
$$

where $I_{[A]}$ is the indicator function of set $A$. Obviously, $y\left(t_{k}\right)=Y\left(t_{k}\right)=y_{k}$ for any integer $k \geq$ 0 ; that is the continuous exponential Euler solution $y(t)$ and the step function $Y(t)$ coincide with the discrete solution at the grid point.

\subsection{Strong Convergence}

In this subsection, we are taking aim at the convergence of exponential Euler method applying to (2.1). To show this, some conditions are imposed to the functions $f$ and $g$ in (2.1). 
Assumption 2.1. Assume that $f$ and $g$ satisfy the globally Lipschitz condition and the linear growth condition, that is, there exist two constants $L_{1}, L_{2}$ such that

$$
\begin{gathered}
|f(t, X)-f(t, Y)|^{2} \vee|g(t, X)-g(t, Y)|^{2} \leq L_{1}|X-Y|^{2} \\
|f(t, X)|^{2} \vee|g(t, Y)|^{2} \leq L_{2}\left(1+|X|^{2}\right)
\end{gathered}
$$

for all $X, Y \in L_{\mathfrak{q}_{0}}^{p}\left(\Omega ; R^{n}\right)$. Furthermore, $f$ and $g$ are supposed to satisfy the following property:

$$
|f(t, X)-f(s, X)|^{2} \vee|g(t, X)-g(s, X)|^{2} \leq L_{3}\left(1+|X|^{2}\right)|t-s|,
$$

where $L_{3}$ is a constant and $t, s \in[0, T]$ with $t>s$.

The following lemma illustrates that the continuous exponential Euler approximate solution (2.4) is bounded in MS sense and the relationship between continuous approximate solution (2.4) and the step function $Y(t)$.

Lemma 2.2. Under Assumption 2.1, there exist two constants $C_{1}, C_{2}$ independent of $h$ such that

$$
\begin{gathered}
\mathbb{E}\left(\sup _{0 \leq t \leq T}|y(t)|^{2}\right) \leq C_{1}, \\
\mathbb{E}\left(|y(t)-Y(t)|^{2}\right) \leq C_{2} h,
\end{gathered}
$$

for any $t \in[0, T]$.

Proof. From (2.4) and the elementary inequality $(a+b+c)^{2} \leq 3\left(a^{2}+b^{2}+c^{2}\right)$, we have

$$
|y(t)|^{2} \leq 3\left[\left|e^{F t} y_{0}\right|^{2}+\left|\int_{0}^{t} e^{F(t-\bar{s})} f(\bar{s}, Y(s)) d s\right|^{2}+\left|\int_{0}^{t} e^{F(t-\bar{s})} g(\bar{s}, Y(s)) d W(s)\right|^{2}\right]
$$

Taking the expectation on both sides and using the Hölder inequality and Doom's martingale inequality yields

$$
\begin{gathered}
\mathbb{E}\left(\sup _{0 \leq s \leq t}|y(s)|^{2}\right) \leq 3\left[\left|e^{F t}\right|^{2} \mathbb{E}\left|y_{0}\right|^{2}+T \mathbb{E} \int_{0}^{t}\left|e^{F(t-\bar{s})}\right|^{2}|f(\bar{s}, Y(s))|^{2} d s\right. \\
\left.+4 \mathbb{E}\left(\int_{0}^{t}\left|e^{F(t-\bar{s})}\right|^{2}|g(\bar{s}, Y(s))|^{2} d s\right)\right] .
\end{gathered}
$$


Letting $M=\max \left\{\left|e^{F T}\right|^{2}, 1\right\}$, by the linear growth condition (2.7), then

$$
\begin{aligned}
& \mathbb{E}\left(\sup _{0 \leq s \leq t}|y(s)|^{2}\right) \\
& \leq 3 M\left[\mathbb{E}\left|y_{0}\right|^{2}+T \int_{0}^{t} \mathbb{E}|f(\bar{s}, Y(s))|^{2} d s+4 E \int_{0}^{t} \mathbb{E}|g(\bar{s}, Y(s))|^{2} d s\right] \\
& \quad \leq 3 M\left[\mathbb{E}\left|y_{0}\right|^{2}+L_{2} T \int_{0}^{t}\left(1+\mathbb{E}|Y(s)|^{2}\right) d s+4 L_{2} \int_{0}^{t}\left(1+\mathbb{E}|Y(s)|^{2} d s\right)\right] \\
& \quad \leq 3 M\left[\mathbb{E}\left|y_{0}\right|^{2}+L_{2} T(T+4)+L_{2}(T+4) \int_{0}^{t}\left(\mathbb{E}|Y(s)|^{2} d s\right)\right] \\
& \quad \leq 3 M\left(\mathbb{E}\left|y_{0}\right|^{2}+L_{2} T(T+4)\right)+3 M L_{2}(T+4) \int_{0}^{t} \mathbb{E}\left(\sup _{0 \leq r \leq s}|y(r)|^{2}\right) d s .
\end{aligned}
$$

Now using the Gronwall inequality yields that

$$
\mathbb{E}\left(\sup _{0 \leq s \leq t}|y(s)|^{2}\right) \leq C_{1}
$$

where $C_{1}=3 M\left(\mathbb{E}\left|y_{0}\right|^{2}+L_{2} T(T+4)\right) e^{3 M L_{2}(T+4) T}$.

From the definition of $Y(t)$ and (2.4), for $t \in\left[t_{k}, t_{k+1}\right)$, we can obtain

$$
y(t)-Y(t)=e^{F\left(t-t_{k}\right)} y_{k}+\int_{t_{k}}^{t} e^{F(t-\bar{s})} f\left(t_{k}, y_{k}\right) d s+\int_{t_{k}}^{t} e^{F(t-\bar{s})} g\left(t_{k}, y_{k}\right) \mathrm{d} W(s)-y_{k} .
$$

Using Hölder inequality gives

$$
\begin{aligned}
& |y(t)-Y(t)|^{2} \\
& \quad \leq 3\left[\left|e^{F\left(t-t_{k}\right)}-I_{n}\right|^{2}\left|y_{k}\right|^{2}+h \int_{t_{k}}^{t}\left|e^{F(t-\bar{s})}\right|^{2}\left|f\left(t_{k}, y_{k}\right)\right|^{2} \mathrm{~d} s+\left|\int_{t_{k}}^{t} e^{F(t-\bar{s})} g\left(t_{k}, y_{k}\right) \mathrm{d} W(s)\right|^{2}\right],
\end{aligned}
$$

where $I_{n}$ is the $n$ dimension identity matrix. Taking the expectation of both sides, we have

$$
\begin{gathered}
\mathbb{E}|y(t)-Y(t)|^{2} \leq 3\left[\left|e^{F\left(t-t_{k}\right)}-I_{n}\right|^{2} \mathbb{E}\left|y_{k}\right|^{2}+h \mathbb{E} \int_{t_{k}}^{t}\left|e^{F(t-\bar{s})}\right|^{2}\left|f\left(t_{k}, y_{k}\right)\right|^{2} d s\right. \\
\left.+\mathbb{E} \int_{t_{k}}^{t}\left|e^{F(t-\bar{s})}\right|^{2}\left|g\left(t_{k}, y_{k}\right)\right|^{2} d s\right] .
\end{gathered}
$$


In view of (2.7), (2.9), and $\left|e^{F\left(t-t_{k}\right)}-I_{n}\right|^{2} \sim O\left(h^{2}\right)$, we can obtain

$$
\begin{aligned}
\mathbb{E}|y(t)-Y(t)|^{2} & \leq 3\left[\left|e^{F\left(t-t_{k}\right)}-I_{n}\right|^{2} C_{1}+h M L_{2}\left(1+C_{1}\right) h+M L_{2}\left(1+C_{1}\right) h\right] \\
& \leq 3 M\left(1+C_{1}\right) L_{2} h+O\left(h^{2}\right) .
\end{aligned}
$$

Therefore,

$$
\mathbb{E}|y(t)-Y(t)|^{2} \leq C_{2} h
$$

where $C_{2}=3 M\left(1+C_{1}\right) L_{2}$.

In the following, we show the convergence result of exponential Euler method for semi-linear SDE (2.1).

Theorem 2.3. Under Assumption 2.1, the numerical solution produced by the exponential Euler method converges to the exact solution of (2.1) in MS sense with the strong order 1/2, that is, there exist a positive constant $C$ such that

$$
\mathbb{E}\left(\sup _{0 \leq t \leq T}|X(t)-y(t)|^{2}\right) \leq C h, \quad \text { as } h \longrightarrow 0
$$

Proof. From (2.2) and (2.4) we know

$$
\begin{aligned}
X(t)-y(t)= & \int_{0}^{t}\left[e^{F(t-s)} f(s, X(s))-e^{F(t-\bar{s})} f(\bar{s}, Y(s))\right] d s \\
& +\int_{0}^{t}\left[e^{F(t-s)} g(s, X(s))-e^{F(t-\bar{s})} g(\bar{s}, Y(s))\right] d W(s), \\
|X(t)-y(t)|^{2} \leq & 2\left\{\left|\int_{0}^{t}\left[e^{F(t-s)} f(s, X(s))-e^{F(t-\bar{s})} f(\bar{s}, Y(s))\right] d s\right|^{2}\right. \\
& \left.+\left|\int_{0}^{t}\left[e^{F(t-s)} g(s, X(s))-e^{F(t-\bar{s})} g(\bar{s}, Y(s))\right] d W(s)\right|^{2}\right\} .
\end{aligned}
$$

By the Hölder inequality and Doom's martingale inequality, we have

$$
\begin{aligned}
\mathbb{E}\left(\sup _{0 \leq r \leq t}|X(r)-y(r)|^{2}\right) \leq 2 & {\left[T \mathbb{E} \int_{0}^{t}\left|e^{F(t-s)} f(s, X(s))-e^{F(t-\bar{s})} f(\bar{s}, Y(s))\right|^{2} d s\right.} \\
& \left.+4 \mathbb{E} \int_{0}^{t}\left|e^{F(t-s)} g(s, X(s))-e^{F(t-\bar{s})} g(\bar{s}, Y(s))\right|^{2} d s\right] .
\end{aligned}
$$


Consider the first argument in (2.23)

$$
\begin{aligned}
& \mathbb{E} \int_{0}^{t}\left|e^{F(t-s)} f(s, X(s))-e^{F(t-\bar{s})} f(\bar{s}, Y(s))\right|^{2} d s \\
& =\mathbb{E} \int_{0}^{t} \mid\left[e^{F(t-s)} f(s, X(s))-e^{F(t-\bar{s})} f(s, X(s))\right]+\left[e^{F(t-\bar{s})} f(s, X(s))-e^{F(t-\bar{s})} f(\bar{s}, X(s))\right] \\
& \quad+\left.\left[e^{F(t-\bar{s})} f(\bar{s}, X(s))-e^{F(t-\bar{s})} f(\bar{s}, Y(s))\right]\right|^{2} d s \\
& \leq 3 \int_{0}^{t}\left|e^{F(t-s)}-e^{F(t-\bar{s})}\right|^{2} \mathbb{E}|f(s, X(s))|^{2} d s+3 \mathbb{E} \int_{0}^{t}\left|e^{F(t-\bar{s})}\right|^{2}|f(s, X(s))-f(\bar{s}, X(s))|^{2} d s \\
& \quad+3 \mathbb{E} \int_{0}^{t}\left|e^{F(t-\bar{s})}\right|^{2}|f(\bar{s}, X(s))-f(\bar{s}, Y(s))|^{2} d s .
\end{aligned}
$$

By Assumption 2.1 and Lemma 2.2, it is obvious that

$$
\begin{aligned}
& \mathbb{E} \int_{0}^{t}\left|e^{F(t-s)} f(s, X(s))-e^{F(t-\bar{s})} f(\bar{s}, Y(s))\right|^{2} d s \\
& \leq 3 L_{2} \int_{0}^{t}\left|e^{F(t-\bar{s})}\right|^{2}\left|e^{F(\bar{s}-s)}-I_{n}\right|^{2}\left[1+\mathbb{E}|X(t)|^{2}\right] d s \\
& \quad+3 L_{3}(s-\bar{s}) M \mathbb{E} \int_{0}^{t}\left[1+|X(s)|^{2}\right] \mathrm{d} s+3 M L_{1} \int_{0}^{t} \mathbb{E}|X(s)-Y(s)|^{2} d s \\
& \leq 3 L_{2} M\left|e^{F h}-I_{n}\right|^{2}\left[1+C_{1}\right] T+3 M L_{3}\left(1+C_{1}\right) T h \\
& \quad+6 L_{1} M \int_{0}^{t} \mathbb{E}\left\{|X(s)-y(s)|^{2}+|y(s)-Y(s)|^{2}\right\} d s \\
& \leq 3 M\left(1+C_{1}\right) T\left[\left|e^{F h}-I_{n}\right|^{2} L_{2}+L_{3} h\right]+6 L_{1} M T C_{2} h+6 L_{1} M \int_{0}^{t} \mathbb{E}|X(s)-y(s)|^{2} d s .
\end{aligned}
$$

This implies

$$
\begin{aligned}
& \mathbb{E} \int_{0}^{t}\left|e^{F(t-s)} f(s, X(s))-e^{F(t-\bar{s})} f(\bar{s}, Y(s))\right|^{2} d s \\
& \quad \leq 3 M\left(1+C_{1}\right) T\left[L_{2}\left|e^{F h}-I_{n}\right|^{2}+L_{3} h\right]+6 L_{1} M C_{2} T h+6 L_{1} M \int_{0}^{t} \mathbb{E}\left(\sup _{0 \leq r \leq s}|X(r)-y(r)|^{2}\right) d s .
\end{aligned}
$$


By the similar procedure, we can observe that

$$
\begin{aligned}
& \mathbb{E} \int_{0}^{t}\left|e^{F(t-s)} g(s, X(s))-e^{F(t-\bar{s})} g(\bar{s}, Y(s))\right|^{2} d s \\
& \quad \leq 3 M\left(1+C_{1}\right) T\left[L_{2}\left|e^{F h}-I_{n}\right|^{2}+L_{3} h\right]+6 L_{1} M C_{2} T h+6 L_{1} M \int_{0}^{t} \mathbb{E}\left(\sup _{0 \leq r \leq s}|X(r)-y(r)|^{2}\right) d s .
\end{aligned}
$$

Substituting (2.26) and (2.27) into (2.23) gives

$$
\begin{aligned}
& \mathbb{E}\left(\sup _{0 \leq r \leq t}|X(r)-y(r)|^{2}\right) \\
& \leq 6 M\left(1+C_{1}\right) T\left[L_{2}\left|e^{F h}-I_{n}\right|^{2}+L_{3} h\right](T+4)+12 L_{1} M C_{2} T(T+4) h \\
& \quad+12 L_{1} M(T+4) \int_{0}^{t} \mathbb{E} \sup _{0 \leq r \leq s}|X(r)-y(r)|^{2} d s .
\end{aligned}
$$

Since $\left|e^{F h}-I_{n}\right| \sim O\left(h^{2}\right)$, we can show the following result by Gronwall inequality:

$$
\mathbb{E}\left(\sup _{0 \leq r \leq t}|X(r)-y(r)|^{2}\right) \leq 6 M T(T+4)\left[L_{3}\left(1+C_{1}\right)+2 L_{1} C_{2}\right] e^{12 L_{1} M(T+4) T} h .
$$

Choosing $C=6 M T(T+4)\left[L_{3}\left(1+C_{1}\right)+2 L_{1} C_{2}\right] e^{12 L_{1} M(T+4) T}$, we can obtain the convergence result for any $0 \leq t \leq T$.

\section{Mean-Square Stability}

In this section, we focus on the MS stability of the exponential Euler as it is applied to scalar semi-linear SDEs. It is significantly helpful to describe the MS stability region of the exponential Euler method. In the following, scalar linear SDE as the test equation is used to calculate the MS stability region.

\subsection{Test Equation}

Consider the test equation

$$
\mathrm{d} y(t)=\lambda y(t) \mathrm{d} t+\mu y(t) d W(t)
$$

where $\lambda, \mu \in \mathbb{R}$ and $W(t)$ is the scalar Wiener process.

It is well known [18] that the solution of (3.1) is MS stable if and only if

$$
2 \lambda+|\mu|^{2}<0
$$


The MS stability region of (3.1) is denoted by $S_{\mathrm{SDE}}=S_{\mathrm{SDE}}(\lambda, \mu)$ and represents the set of parameter values for which the equilibrium solution of (3.1) is MS stable. The exponential Euler method for test equation (3.1) leads to the following type:

$$
\begin{aligned}
X_{n+1} & =e^{\lambda h} X_{n}+e^{\lambda h} \mu X_{n} \Delta W_{n} \\
& =e^{\lambda h} X_{n}+e^{\lambda h} \mu X_{n} \sqrt{h} Z \\
& =e^{\lambda h}(1+\mu \sqrt{h} Z) X_{n}
\end{aligned}
$$

where $X_{n}$ is the approximation of $y\left(t_{n}\right)$ with $X_{0}=y(0)$. Now we define MS stability region for the numerical method applied to (3.1). The notations and definitions are similar to those in [8].

Definition 3.1. If the adaptation of a numerical method to (3.1) leads to a numerical process of the following type:

$$
X_{n+1}=R(h, \lambda, \mu, Z) X_{n}
$$

$\bar{R}(h, \lambda, \mu):=\mathbb{E}|R(h, \lambda, \mu, Z)|^{2}$ is called the MS stability function of the numerical solution. Furthermore, if $\bar{R}(h, \lambda, \mu)<1$, the numerical method is MS stable and the region of parameter values that satisfy $\bar{R}(h, \lambda, \mu)<1$ is called the MS stability region of the numerical method, where $Z \sim N(0,1)$.

The MS stability regions of EM method and exponential Euler method are denoted by $S_{\mathrm{EM}}, S_{\mathrm{EE}}$, respectively.

Definition 3.2. The exponential Euler method described by (2.3) is mean-square $A$-stable if for all $h$,

$$
S_{\mathrm{SDE}} \subseteq S_{\mathrm{EE}}
$$

Higham [19] proposed that the MS stability region for EM method is $S_{\mathrm{EM}}=\{(p, q) \mid 0<$ $q<-p(p+2)\}$, where $p=\lambda h$ and $q=|\mu|^{2} h$. According to (3.2), the SDE (3.1) is MS stable only and if only the pair of parameters $\lambda$ and $\mu$ belong to the region of $S_{\mathrm{SDE}}=\{(p, q) \mid 0<q<-2 p\}$.

The MS stability region of exponential Euler method for (3.1) is given in the following theorem. By the comparing with the Euler method and stochastic Theta method, it is observed that the exponential Euler method as an explicit numerical method has desired property.

Theorem 3.3. The mean-square stability region of exponential Euler method for (3.1) is

$$
S_{\mathrm{EE}}=\left\{(p, q) \mid 0<q<e^{-2 p}-1\right\},
$$

where $p=\lambda$ h and $q=|\mu|^{2} h$. 
Proof. From (3.3), by using $\mathbb{E} Z=0$ and $\mathbb{E} Z^{2}=1$, the MS stability function of exponential Euler method is

$$
\begin{aligned}
\bar{R}(h, \lambda, \mu, Z) & =\mathbb{E}|R(h, \lambda, \mu, Z)|^{2} \\
& =\mathbb{E}\left|e^{\lambda h}(1+\mu \sqrt{h} Z)\right|^{2} \\
& =e^{2 \lambda h}\left(1+|\mu|^{2} h\right) .
\end{aligned}
$$

Letting $p=\lambda h$ and $q=|\mu|^{2} h$,

$$
\bar{R}(h, \lambda, \mu, Z)=e^{2 p}(1+q)
$$

If $e^{2 p}(1+q)<1$, exponential Euler method for (3.1) is MS stable, that is,

$$
q<e^{-2 p}-1
$$

Clearly, the MS stability region of exponential Euler method is $S_{\mathrm{EE}}=\left\{(p, q) \mid 0<q<e^{-2 p}-\right.$ $1\}$.

Remark 3.4. From the inequality $-p(p+2)<-2 p<e^{-2 p}-1$, we can find that MS stability region of exponential Euler method contains that of EM method and contains MS stability region of the exact solution for (3.1), that is,

$$
S_{\mathrm{EM}} \subset S_{\mathrm{SDE}} \subset S_{\mathrm{EE}}
$$

According to the Definition 3.2, the exponential Euler method is mean-square $A$-stable.

Remark 3.5. In [9] the MS stability region of stochastic Theta method is denoted by $S_{\mathrm{STM}}(\theta, h)$ and had the conclusions that $S_{\mathrm{STM}}(\theta, h) \subset S_{\mathrm{SDE}}$ if $0 \leq \theta<1 / 2$ and $S_{\mathrm{SDE}} \subset S_{\mathrm{STM}}(\theta, h)$ if $1 / 2<\theta<1$. We know that stochastic Theta method is MS stable if and only if

$$
\frac{|1+(1-\theta) h \lambda|^{2}+h|\mu|^{2}}{|1-\theta h \lambda|^{2}}<1 .
$$

Letting $p=\lambda h$ and $q=|\mu|^{2} h$; therefore, the stochastic Theta method is MS stable if and only if $q<(2 \theta-1) p^{2}-2 p$. Derived from the inequality $(2 \theta-1) p^{2}-2 p<e^{-2 p}-1$, we find that

$$
\begin{array}{ll}
S_{\mathrm{STM}}(\theta, h) \subset S_{\mathrm{SDE}} \subset S_{\mathrm{EE}} & \text { if } 0 \leq \theta<\frac{1}{2} \\
S_{\mathrm{SDE}} \subset S_{\mathrm{STM}}(\theta, h) \subset S_{\mathrm{EE}} & \text { if } \frac{1}{2}<\theta<1 .
\end{array}
$$




\subsection{Scalar Semilinear SDEs}

This subsection presents new result on the MS exponentially stability for scalar semi-linear stochastic differential equation

$$
d X(t)=[a X(t)+f(t, X(t))] d t+g(t, X(t)) d W(t),
$$

with initial value $X(0)=X_{0}$, where $a<0$ is the linear argument of the drift coefficient.

We have proved that the exponential Euler approximation solution preserves the MS exponential stability of the exact solution for any stepsize $h$ under the following conditions.

Assumption 3.6. Assume that there exist a positive constant $K$ such that

$$
|f(t, X)|^{2} \vee|g(t, X)|^{2} \leq K|X|^{2} .
$$

The condition (3.14) implies $f(t, 0)=0, g(t, 0)=0$ and ensures that the analytical solution will never reach the origin with probability one.

Definition 3.7 (see [20]). The solution of (3.13) is said to be exponentially stable in MS sense if there is a pair of positive constants $\gamma$ and $C$ such that

$$
\mathbb{E}|X(t)|^{2} \leq C\left|X_{0}\right|^{2} e^{-\gamma t}
$$

Lemma 3.8. Under Assumption 3.6, if

$$
\rho:=2 a+2 \sqrt{K}+K<0,
$$

the analytic solution of (3.13) is exponentially stable in MS sense, that is,

$$
\mathbb{E}|X(t)|^{2} \leq \sqrt{2}\left(1+\mathbb{E}\left|X_{0}\right|^{2}\right) e^{2 \rho t}
$$

This lemma can be proved in the similar way as Theorem 4.1 proved in [20]. We can obtain that if $\rho<0$, the analytic solution is exponentially stable in MS sense.

Now the original result about the MS exponential stability of exponential Euler method is given in the following theorem.

Theorem 3.9. If (3.14) and (3.16) hold, then for any stepsize $h>0$, the exponential Euler method for (3.13) is exponentially stable in MS sense, that is,

$$
\lim _{n \rightarrow \infty} \frac{1}{n h} \ln \mathbb{E}\left|y_{n}\right|^{2} \leq \beta<0
$$

where $\beta=2 a+(1 / h) \ln \left(1+K h^{2}+K h+2 \sqrt{K} h\right)$. 
Proof. The adaptation of exponential Euler method to (3.13) leads to a numerical process of the following type:

$$
y_{n+1}=e^{a h} y_{n}+e^{a h} f\left(t_{n}, y_{n}\right) h+e^{a h} g\left(t_{n}, y_{n}\right) \Delta W_{n}
$$

where $\Delta W_{n} \sim N(0, h)$. Clearly,

$$
\begin{aligned}
\left|y_{n+1}\right|^{2}= & e^{2 a h}\left|y_{n}\right|^{2}+e^{2 a h}\left|f\left(t_{n}, y_{n}\right)\right|^{2} h^{2}+e^{2 a h}\left|g\left(t_{n}, y_{n}\right)\right|^{2} \Delta W_{n}^{2} \\
& +2 e^{2 a h}\left\langle y_{n}, f\left(t_{n}, y_{n}\right)\right\rangle h+2 e^{2 a h}\left\langle y_{n}, g\left(t_{n}, y_{n}\right)\right\rangle \Delta W_{n} \\
& +2 e^{2 a h}\left\langle f\left(t_{n}, y_{n}\right), g\left(t_{n}, y_{n}\right)\right\rangle h \Delta W_{n} .
\end{aligned}
$$

Taking the conditional expectation of both sides yields

$$
\begin{aligned}
\mathbb{E}\left(\left|y_{n+1}\right|^{2} \mid \mathcal{F}_{n h}\right)=e^{2 a h}\left|y_{n}\right|^{2} I_{\left\{y_{n} \neq 0\right\}} \mathbb{E}\{ & \left(1+\frac{\left|f\left(t_{n}, y_{n}\right)\right|^{2} h^{2}}{\left|y_{n}\right|^{2}}+\frac{\left|g\left(t_{n}, y_{n}\right)\right|^{2}}{\left|y_{n}\right|^{2}} \Delta W_{n}^{2}\right. \\
& +2 \frac{\left\langle y_{n}, f\left(t_{n}, y_{n}\right)\right\rangle}{\left|y_{n}\right|^{2}} h+2 \frac{\left\langle y_{n}, g\left(t_{n}, y_{n}\right)\right\rangle}{\left|y_{n}\right|^{2}} \Delta W_{n} \\
& \left.\left.+2 \frac{\left\langle f\left(t_{n}, y_{n}\right), g\left(t_{n}, y_{n}\right)\right\rangle}{\left|y_{n}\right|^{2}} h \Delta W_{n}\right) \mid \mathcal{F}_{k h}\right\} .
\end{aligned}
$$

Note that $\Delta W_{n}$ is independent of $\mathcal{F}_{n h}, \mathbb{E}\left(\Delta W_{n} \mid \mathcal{F}_{n h}\right)=\mathbb{E}\left(\Delta W_{n}\right)=0$ and $\mathbb{E}\left(\Delta W_{n}^{2} \mid \mathcal{F}_{n h}\right)=$ $\mathbb{E}\left(\Delta W_{n}^{2}\right)=h$. We can obtain that

$$
\begin{aligned}
\mathbb{E}\left(\left|y_{n+1}\right|^{2} \mid \mathcal{F}_{n h}\right)=e^{2 a h}\left|y_{n}\right|^{2} I_{\left\{y_{n} \neq 0\right\}}( & +\frac{\left|f\left(t_{n}, y_{n}\right)\right|^{2} h^{2}}{\left|y_{n}\right|^{2}}+\frac{\left|g\left(t_{n}, y_{n}\right)\right|^{2}}{\left|y_{n}\right|^{2}} h \\
& \left.+2 \frac{\left\langle y_{n}, f\left(t_{n}, y_{n}\right)\right\rangle}{\left|y_{n}\right|^{2}} h\right) .
\end{aligned}
$$

From the linear condition (3.14), we obtain that

$$
\mathbb{E}\left(\left|y_{n+1}\right|^{2} \mid \mathcal{F}_{n h}\right) \leq e^{2 a h}\left|y_{n}\right|^{2} I_{\left\{y_{n} \neq 0\right\}}\left(1+K h^{2}+K h+2 \sqrt{K} h\right) .
$$

Taking expectations on both sides yields

$$
\mathbb{E}\left|y_{n+1}\right|^{2} \leq \mathbb{E}\left|y_{n}\right|^{2} e^{2 a h}\left(1+K h^{2}+K h+2 \sqrt{K} h\right) .
$$

If

$$
2 a h+\ln \left(1+K h^{2}+k h+2 \sqrt{K} h\right)<0,
$$


exponential Euler method is exponentially MS stable. Subsequently, we proof that (3.25) holds under the condition (3.14) and (3.16).

Recall the inequality $1+x+x^{2} / 2 !+x^{3} / 3 !<e^{x}$. If

$$
\left(1+K h^{2}+K h+2 \sqrt{K} h\right)<1-2 a h+\frac{(-2 a h)^{2}}{2 !}+\frac{(-2 a h)^{3}}{3 !}
$$

then $\left(1+K h^{2}+k h+2 \sqrt{K} h\right)<e^{-2 a h}$ and (3.25) holds. Simplifying the (3.26), we have

$$
\frac{4}{3} a^{3} h^{2}+\left(K-2 a^{2}\right) h+2 \sqrt{K}+K+2 a<0 .
$$

Let $f(h)=(4 / 3) a^{3} h^{2}+\left(K-2 a^{2}\right) h+2 \sqrt{K}+2 a+K$. From $a<0$ and $\rho<0$, it is easy to observe that $f^{\prime}(h)=(8 / 3) a^{3} h+\left(K-2 a^{2}\right)<0$ when $h>0$ and $f(0)=\rho<0$. Hence (3.26) holds and this implies (3.25) always holds when $h>0$.

From(3.24), it follows that

$$
\mathbb{E}\left|y_{n}\right|^{2} \leq \mathbb{E}\left|y_{0}\right|^{2} e^{2 a h n}\left(1+K h^{2}+K h+2 \sqrt{K} h\right)^{n}
$$

Then

$$
\lim _{n \rightarrow \infty} \frac{1}{n h} \ln \mathbb{E}\left|y_{n}\right|^{2}<\beta,
$$

where $\beta=2 a+(1 / h) \ln \left(1+K h^{2}+K h+2 \sqrt{K} h\right)$. From (3.25), we can obtain that $\beta<0$ for any stepsize $h>0$.

Remark 3.10. If $2 a+2 \sqrt{K}+K<0$, the analytic solution of (3.13) is exponentially stable in MS sense. Under the same conditions, the numerical solution of exponential Euler method can preserve the exponential stable in MS sense for any stepsize $h>0$, that is, the stability of exponential Euler method for (3.13) has no limitation to the stepsize $h$.

\section{Numerical Experiments}

In this section, several numerical experiments are given to verify the conclusions of convergence and MS stability of exponential Euler method for semi-linear stochastic differential equations.

\subsection{Strong Convergence of Exponential Euler Method}

In order to make the notion of convergence precise, we must decide how to measure their difference. Using $\mathbb{E}\left|y_{n}-X\left(\tau_{n}\right)\right|$ leads to the concept of strong convergence [19]. 


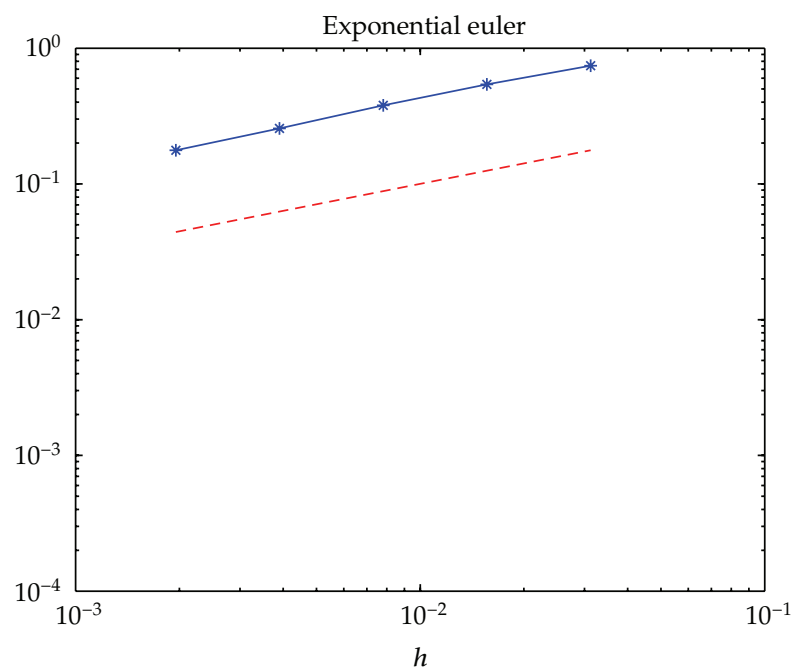

Figure 1: Numerical approximation for strong convergence order of exponential Euler method.

Definition 4.1. A method is said to have strong order of convergence equal to $1 / 2$ if there exists a constant $C$, such that

$$
e(h):=\mathbb{E}\left|y_{n}-X(\tau)\right| \leq C h^{1 / 2},
$$

for any sufficiently small stepsize $h$ and fixed $\tau=n h \in[0, T]$.

The parameter $\lambda=2, \mu=1$ and $X_{0}=1$ is used to look at the strong convergence of exponential Euler method for (3.1). We compute 5000 different discrete Brownian paths over $[0,1]$ with $\delta=2^{-9}$. For each path, exponential Euler method is applied with differential stepsizes: $h=2^{p-1} \delta$ for $1 \leq p \leq 5$. We denote by $y_{k, p}$ the value of $k$ th generated trajectory of numerical solution at the endpoint with $h=2^{p-1} \delta$ for $1 \leq p \leq 5$ and by $X_{k, p}$ the corresponding value of exact solution. It is easy to obtain the analytical solution of (3.1). The average errors

$$
e(h)=\frac{1}{5000} \sum_{k=1}^{5000}\left|y_{k, p}-X_{k, p}\right|
$$

at the endpoint over 5000 sample paths are approximation for $h=2^{p-1} \delta, 1 \leq p \leq 5$. We plot the approximation to $e(h)$ against $h$ in blue on a log-log scale in Figure 1. For reference, a dashed red line of slope one-half is added. In Figure 1, we can see that the slopes of the two curves appear to match well.

If the inequality (4.1) holds with approximate equality, taking logs of both sides,

$$
\log e(h) \approx \log C+\frac{1}{2} \log h .
$$

Furthermore, we see that the slope of the curve appears to $1 / 2$. A least-squares power law fit produces the slope $=0.5218$, residual $=0.0435$ of the blue curve in Figure 1 . This suggests 


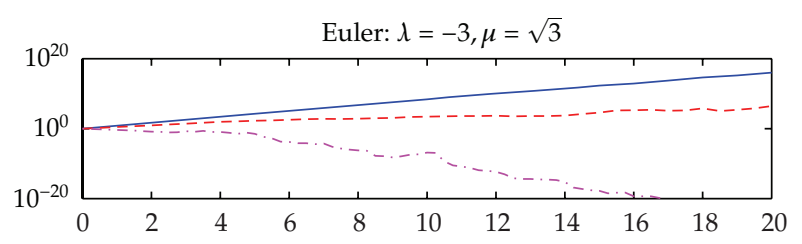

(a)

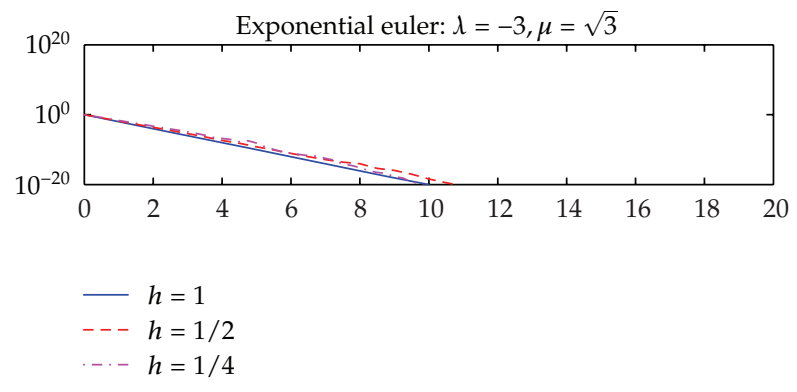

(b)

Figure 2: The numerical solutions with $\lambda=-3, \mu=\sqrt{3}$.

that (4.1) is valid. Therefore, our results are consistent with a strong order of exponential Euler method equal to $1 / 2$ from numerical experience.

\subsection{Mean-Square Stability Region}

Consider the linear scaler stochastic differential equation

$$
\mathrm{d} X(t)=\lambda X(t) \mathrm{d} t+\mu X(t) \mathrm{d} W(t), \quad X(0)=X_{0}
$$

To examine MS stability of exponential Euler method, we solve (4.4) with $X_{0}=1$ over $[0,20]$ for two parameters sets. The first set is $\lambda=-3$ and $\mu=\sqrt{3}$. These values satisfy (3.2), hence the problem is MS stable. Firstly, We apply EM method over 50000 discrete Brownian paths for the three differential stepsizes: $h=1, h=1 / 2, h=1 / 4$. Secondly, we apply exponential Euler method with the same stepsizes. Figure 2 depicts the plot of the sample average of $y_{j}^{2}$ against $t_{j}=j h$. Note that the vertical axis is logarithmically scaled.

In the upper curves of Figure $2, h=1$ and $h=1 / 2$ curves increase with $t$ while the $h=1 / 4$ curve decays toward zero. However, in the lower curves of Figure 2, all the curves decay toward zero whether $h=1, h=1 / 2$, or $h=1 / 4$. This implies that the MS stability region of exponential Euler method contains that of EM method.

Next, we use the parameter set $\lambda=-3$ and $\mu=3$. It is observed that (4.4) is not MS stable. The upper curves of Figure 3 are approximated by the EM method and the lower curves in Figure 3 are approximated by the exponential Euler method. The curves in the upper picture increase with $t$, while all the curves in the lower picture decrease toward zero. This implies that the MS stability region of exponential Euler method contains the MS region of test equation (4.4). 


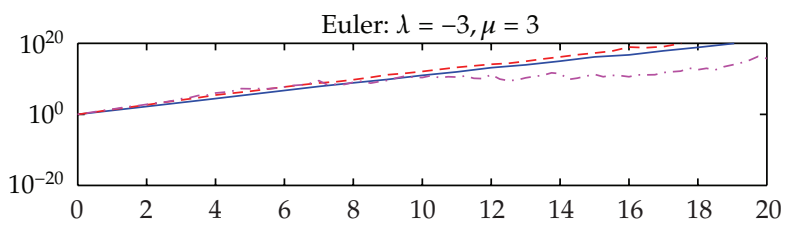

(a)

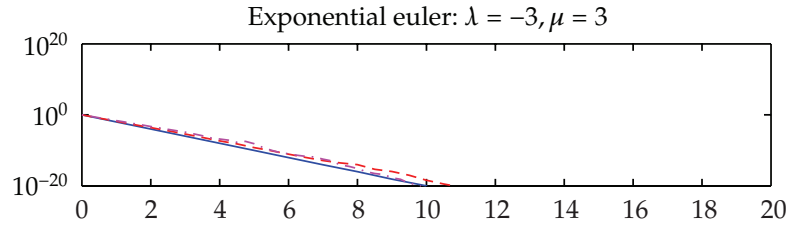

- $h=1$

$--h=1 / 2$

$-\cdot h=1 / 4$

(b)

Figure 3: The numerical solutions with $\lambda=-3, \mu=3$.

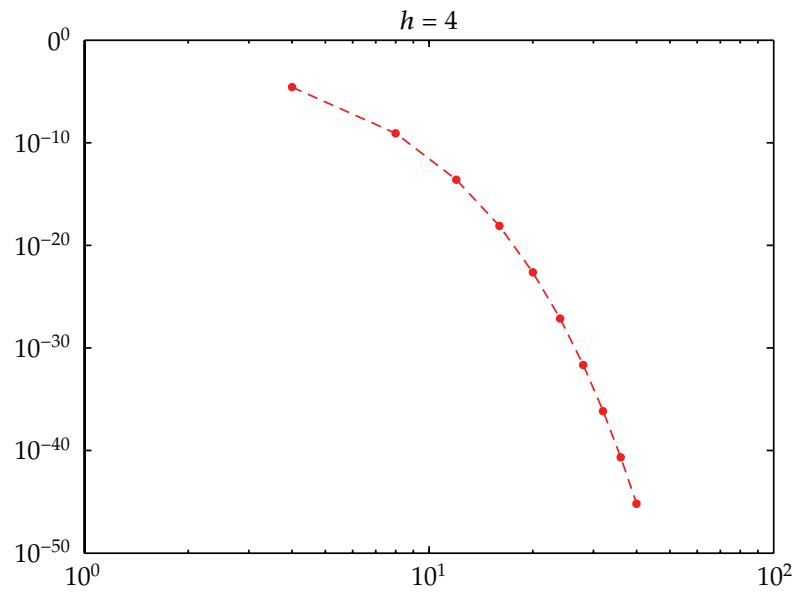

Figure 4: Exponential Euler method.

\subsection{Mean-Square Exponential Stability}

Consider the scalar semi-linear stochastic differential equation:

$$
d x(t)=(-3 x(t)+\sin (x(t))) d t+x(t) d W(t) .
$$

It is easy to verify that (4.5) has the properties of (3.14) and (3.16). According to Theorem 3.9, the problem is exponentially stable in MS sense. To test MS exponential stability, we solve (4.5) with $X_{0}=1$ over $[0,100]$. We apply the exponential Euler method and EM method, respectively, over 5000 different discrete Brownian paths with different large stepsize $h=$ $4, h=8$, and the average over 5000 sample paths is approximated to $\left|y_{n}\right|^{2}$. 


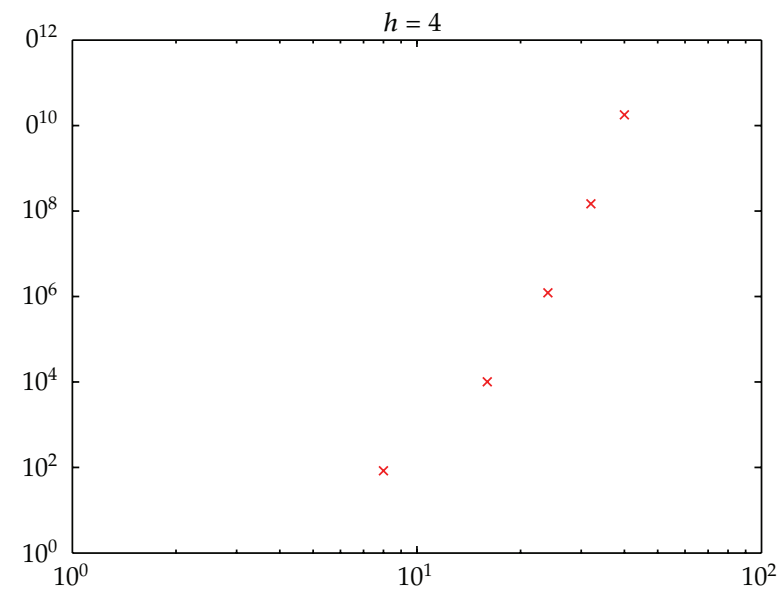

Figure 5: EM method.

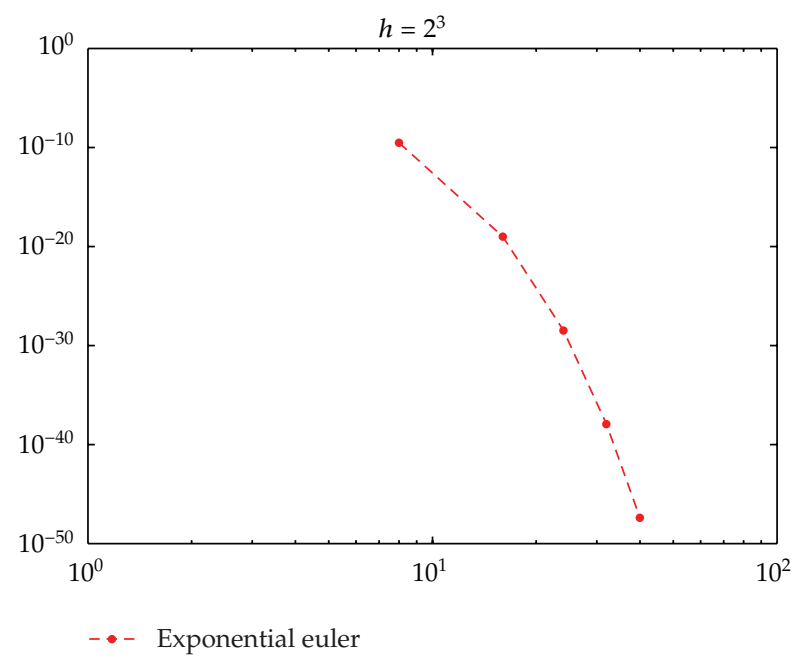

Figure 6: Exponential Euler method.

The solutions of exponential Euler method and EM method with $h=4$ can be found in Figures 4 and 5. We can find that the curve in Figure 4 decreases significantly while the curve in Figure 5 increases sharply with $t$. It is observed that the exponential Euler method can preserve the MS exponential stability, but EM method does not have this property when $h=4$. Figures 6 and 7 demonstrate numerical solutions of the two methods when stepsize $h=$ $2^{3}$ and the similar result can be obtained. Hence exponential Euler approximation solution shares the MS exponential stability of the exact solution.

From Figures 5 and 7, it is manifest that EM method does not preserve the stability with $h=2^{2}$ and $h=2^{3}$. 


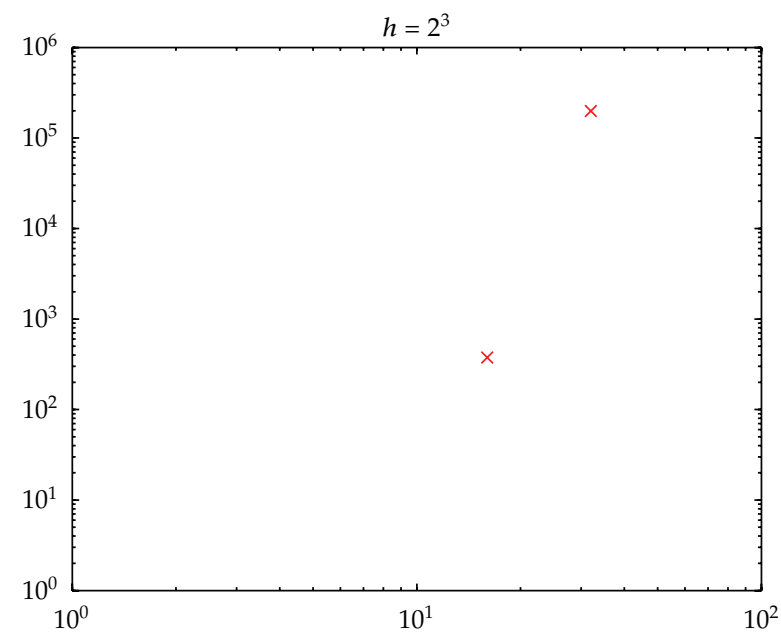

Figure 7: EM method.

\section{Conclusions}

The classical explicit numerical methods for SDEs such as EM method and Milstein method and the semi-implicit method such as stochastic Theta method $(0 \leq \theta<1 / 2)$ usually have limitations to the stepsize $h$, and the stability results are given for sufficient small stepsize $h$. In this paper, the exponential Euler method is extended to semi-linear SDEs and we proved that the stability results have fewer restrictions of stepsize and preserve the stability of SDEs.

It is proved that under the conditions where scalar semi-linear SDEs is MS exponentially stable, the exponential Euler method can preserve the MS stability for all stepsize $h>0$. For scaler linear test equation, the MS stable region of exponential Euler method is calculated, and it is observed that the MS stable region of exponential Euler method contains that of EM method and stochastic Theta method $(0 \leq \theta<1)$ and also contains that of the scalar linear test equation. According to Definition 3.2, exponential Euler method is MS A-stable.

In this paper, the scalar Wiener process is considered and the corresponding results can be generalized to the multidimensional Winer process.

The MS exponential stability is investigated for scalar semi-linear SDEs in this paper. For $n$-dimensional SDEs $(n \geq 2)$,

$$
\begin{aligned}
d X(t) & =f(X(t)) d t+g(X(t)) d W(t), \\
X(0) & =X_{0},
\end{aligned}
$$

whether exponential Euler method can be applied to obtain the numerical solution is our future work.

\section{Acknowledgment}

This paper is supported by the Fundamental Research Funds for the Central Universities (HIT.NSRIF.2013081). 


\section{References}

[1] K. Burrage, P. M. Burrage, and T. Tian, "Numerical methods for strong solutions of stochastic differential equations: an overview," Proceedings of The Royal Society of London A, vol. 460, no. 2041, pp. 373-402, 2004.

[2] P. E. Kloeden and E. Platen, Numerical Solution of Stochastic Differential Equations, Springer, Berlin, Germany, 1992.

[3] G. N. Milstein and M. Tretyakov, Stochastic Numerics for Mathematical Physics, Springer, Berlin, Germany, 2004.

[4] D. J. Higham, X. Mao, and C. Yuan, "Almost sure and moment exponential stability in the numerical simulation of stochastic differential equations," SIAM Journal on Numerical Analysis, vol. 41, no. 2, pp. 592-609, 2007.

[5] S. Pang, F. Deng, and X. R. Mao, "Almost sure and moment exponential stability of Euler-Maruyama discretizations for hybrid stochastic differential equations," Journal of Computational and Applied Mathematics, vol. 213, no. 1, pp. 127-141, 2008.

[6] W. R. Cao, M. Z. Liu, and Z. C. Fan, "MS-stability of the Euler-Maruyama method for stochastic differential delay equations," Applied Mathematics and Computation, vol. 159, no. 1, pp. 127-135, 2004.

[7] Y. Saito and T. Mitsui, "Mean-square stability of numerical schemes for stochastic differential systems," Vietnam Journal of Mathematics, vol. 30, pp. 551-560, 2002.

[8] E. Buckwar and C. Kelly, "Towards a systematic linear stability analysis of numerical methods for systems of stochastic differential equations," SIAM Journal on Numerical Analysis, vol. 48, no. 1, pp. 298-321, 2010.

[9] D. J. Higham, "Mean-square and asymptotic stability of the stochastic theta method," SIAM Journal on Numerical Analysis, vol. 38, no. 3, pp. 753-769, 2000.

[10] B. V. Minchev and W. M. Wright, "A review of exponential integrators for first order semi-linear problems," Tech. Rep. 2/05, Department of Mathematics, NTNU, 2005.

[11] J. D. Lawson, "Generalized Runge-Kutta processes for stable systems with large Lipschitz constants," SIAM Journal on Numerical Analysis, vol. 4, pp. 372-380, 1967.

[12] A. Ostermann, M. Thalhammer, and W. Wright, "A class of explicit exponential general linear methods," BIT Numerical Mathematics, vol. 46, no. 2, pp. 409-431, 2006.

[13] M. Hochbruck and A. Ostermann, "Explicit exponential Runge-Kutta methods for semilinear parabolic problems," SIAM Journal on Numerical Analysis, vol. 43, no. 3, pp. 1069-1090, 2005.

[14] S. Maset and M. Zennaro, "Unconditional stability of explicit exponential Runge-Kutta methods for semi-linear ordinary differential equations," Mathematics of Computation, vol. 78, no. 266, pp. 957-967, 2009.

[15] X. R. Mao, Y. Shen, and A. Gray, "Almost sure exponential of backward Euler-Maruyama discretization for differential equations," Journal of Computational and Applied Mathematics, vol. 235, pp. 1213-1226, 2010.

[16] Z. Y. Wang and C. J. Zhang, "An analysis of stability of Milstein method for stochastic differential equations with delay," Computers $\mathcal{E}$ Mathematics with Applications, vol. 51, no. 9-10, pp. 1445-1452, 2006.

[17] M. Kunze and J. Neerven, "Approximating the coefficients in semilinear stochastic partial differential equations," Journal of Evolution Equations, vol. 11, no. 3, pp. 577-604, 2011.

[18] Y. Saito and T. Mitsui, "Stability analysis of numerical schemes for stochastic differential equations," SIAM Journal on Numerical Analysis, vol. 33, no. 6, pp. 2254-2267, 1996.

[19] D. J. Higham, "An algorithmic introduction to numerical simulation of stochastic differential equations," SIAM Review, vol. 43, no. 3, pp. 525-546, 2001.

[20] X. R. Mao, Stochastic Differential Equations and Application, Horwood, New York, NY, USA, 1997. 


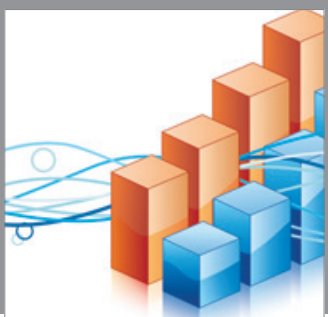

Advances in

Operations Research

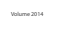

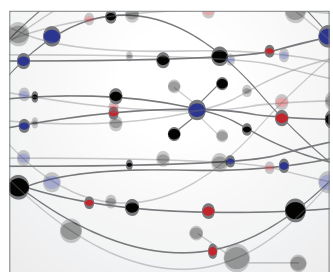

\section{The Scientific} World Journal
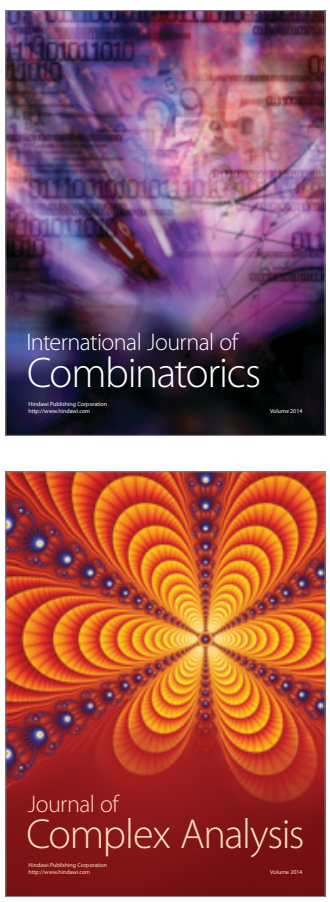

International Journal of

Mathematics and

Mathematical

Sciences
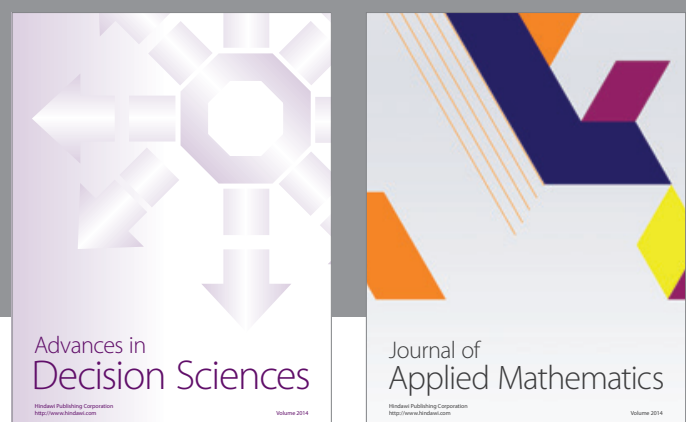

Journal of

Applied Mathematics
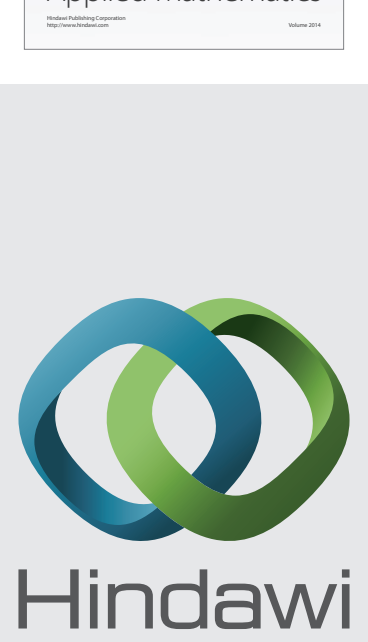

Submit your manuscripts at http://www.hindawi.com
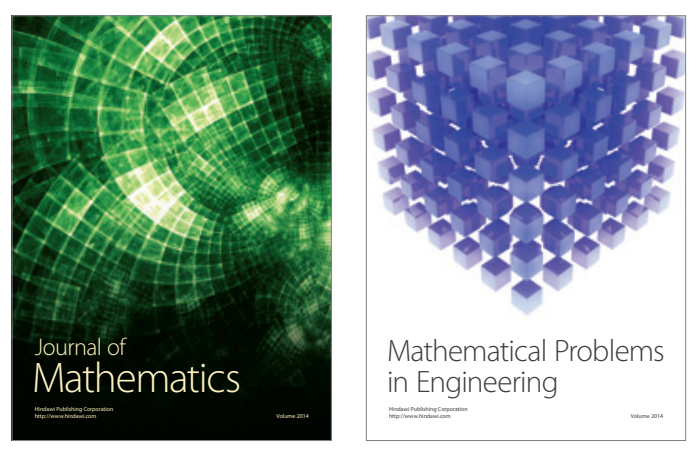

Mathematical Problems in Engineering
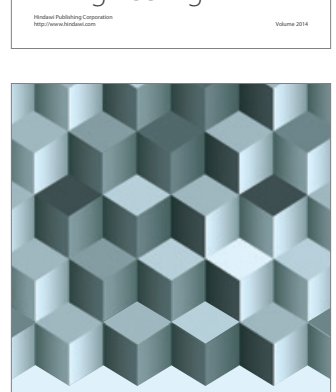

Journal of

Function Spaces
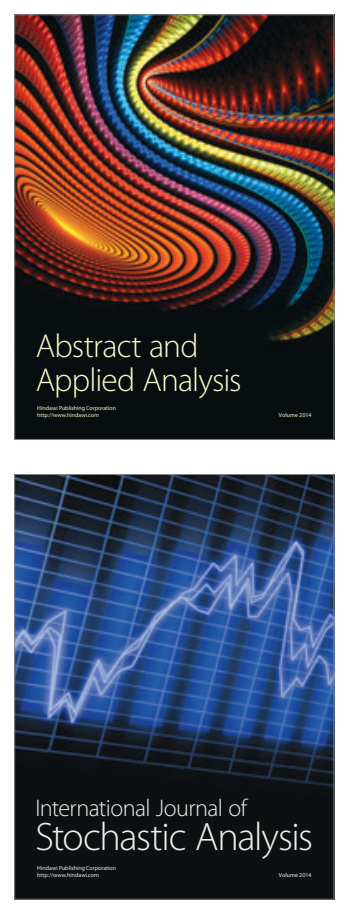

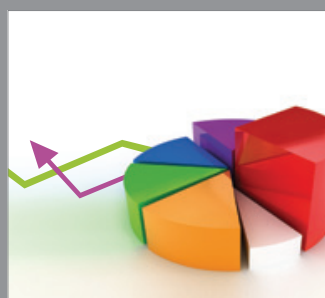

ournal of

Probability and Statistics

Promensencen
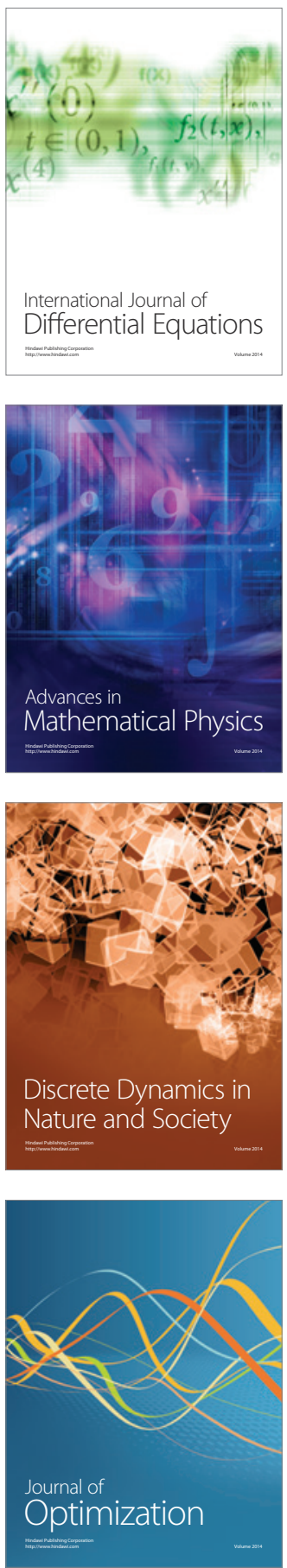